\title{
Suppression of Neural Responses to Nonoptimal Stimuli Correlates With Tuning Selectivity in Macaque V1
}

\author{
D. L. RINGACH, ${ }^{1-3}$ C. E. BREDFELDT, ${ }^{2}$ R. M. SHAPLEY ${ }^{4}$ AND M. J. HAWKEN ${ }^{4}$ \\ ${ }^{1}$ Department of Neurobiology, ${ }^{2}$ Department of Psychology, and ${ }^{3}$ Brain Research Institute, University of California, Los \\ Angeles, California 90095; and ${ }^{4}$ Center for Neural Science, New York University, New York, New York 10003
}

Received 25 July 2001; accepted in final form 15 October 2001

\begin{abstract}
Ringach, D. L., C. E. Bredfeldt, R. M. Shapley, and M. J. Hawken. Suppression of neural responses to nonoptimal stimuli correlates with tuning selectivity in macaque V1. J Neurophysiol 87: 1018-1027, $2002 ; 10.1152 /$ jn.00614.2001. Neural responses in primary visual cortex (area V1) are selective for the orientation and spatial frequency of luminance-modulated sinusoidal gratings. Selectivity could arise from enhancement of the cell's response by preferred stimuli, suppression by nonoptimal stimuli, or both. Here, we report that the majority of V1 neurons do not only elevate their activity in response to preferred stimuli, but their firing rates are also suppressed by nonoptimal stimuli. The magnitude of suppression is similar to that of enhancement. There is a tendency for net response suppression to peak at orientations near orthogonal to the optimal for the cell, but cases where suppression peaks at oblique orientations are observed as well. Interestingly, selectivity and suppression correlate in V1: orientation and spatial frequency selectivity are higher for neurons that are suppressed by nonoptimal stimuli than for cells that are not. This finding is consistent with the idea that suppression plays an important role in the generation of sharp cortical selectivity. We show that nonlinear suppression is required to account for the data. However, the precise structure of the neural circuitry generating the suppressive signal remains unresolved. Our results are consistent with both feedback and (nonlinear) feed-forward inhibition.
\end{abstract}

\section{N T R O D U C T I O N}

The cortical representation of the retinal image undergoes profound transformations as it progresses along the visual pathway. Cells in primary visual cortex are tuned for local attributes of the image, such as stimulus orientation, spatial frequency, and color, among other dimensions (De Valois et al. 1982; Hubel and Wiesel 1962, 1968; Movshon et al. 1978). Understanding how such selectivity is generated in V1 requires separating the relative contributions of the feedforward circuitry and intracortical mechanisms.

An important question is the role that intracortical inhibition plays in the generation of stimulus selectivity (Anderson et al. 2000; Benevento et al. 1972; Blakemore and Tobin 1972; Bonds 1989; De Valois and Tootell 1983; Ferster and Miller 2000; Hata et al. 1988; Morrone et al. 1982; Nelson and Frost 1978; Nelson 1991; Ramoa et al. 1986; Ringach et al. 1997b; Sillito et al. 1980; Sompolinsky and Shapley 1997; Volgushev et al. 1993). There is an on-going debate regarding the role of inhibition in establishing sharp selectivity for orientation. On

Address for reprint requests: D. L. Ringach, Dept. of Neurobiology and Psychology, Franz Hall, Rm. 8441B, University of California, Los Angeles, CA 90095-1563 (E-mail: dario@ucla.edu). one hand, some studies suggest that inhibition has no role in sharpening tuning selectivity. This claim appears to be supported by the following findings: the tuning of excitation and inhibition, recorded in layer 4 cortical neurons intracellularly, appear to be very similar (Carandini and Ferster 2000; Ferster 1986); the tuning of a V1 cell's membrane potential modulation in response to drifting grating stimuli remains unchanged when the cortex is inactivated by cooling or by electrical stimulation (Chung and Ferster 1998; Ferster et al. 1996); and neurons retain their tuning selectivity when inhibition is blocked intracellularly (Nelson et al. 1994). On the other hand, several studies suggest that suppression plays a critical role in establishing sharp selectivity for orientation. Some of the findings that support this view are response enhancement and suppression in V1 have different bandwidths, with suppression being more broadly tuned than enhancement (Blakemore and Tobin 1972; Bonds 1989; Burr et al. 1981; Monier et al. 2000; Morrone et al. 1982; Nelson and Frost 1978; Nelson 1991; Ringach et al. 1997a); blocking inhibition pharmacologically leads to a broadening in V1 tuning selectivity (Allison and Bonds 1994; Allison et al. 1995, 1996; Crook et al. 1998; Hata et al. 1988; Sato et al. 1996; Sillito et al. 1980); and theoretical work indicates that broadly tuned inhibition could act as a mechanism to suppress the response of the cell to nonoptimal stimuli and sharpen selectivity (Ben-Yishai et al. 1995; McLaughlin et al. 2000; Pugh et al. 2000; Somers et al. 1995). There is also a similar lack of agreement about the role of inhibition in spatial frequency tuning. Some authors report the absence of response suppression at nonoptimal frequencies (Ramoa et al. 1986), while others find a substantial amount of suppression (Bauman and Bonds 1991; De Valois and Tootell 1983).

We studied the steady-state and dynamical tuning properties of neurons in the joint orientation and spatial frequency domain (the Fourier plane). The dynamics of orientation and spatial frequency were measured using a new reverse correlation method that was an improvement on methods used previously to measure orientation tuning dynamics (Ringach et al. 1997b). The pattern of response enhancement and suppression in the Fourier plane revealed that the responses of the most highly selective neurons are almost always suppressed by nonoptimal stimuli. This correlation between high selectivity and suppres-

\footnotetext{
The costs of publication of this article were defrayed in part by the payment of page charges. The article must therefore be hereby marked "advertisement" in accordance with 18 U.S.C. Section 1734 solely to indicate this fact.
} 
sion indicates that intracortical inhibition probably plays an important role in the generation of sharp stimulus selectivity.

\section{METHODS}

Acute experiments were performed on adult Old World monkeys (Macaca fascicularis) in compliance with National Institutes of Health and University of California Los Angeles/Animal Research Committee guidelines. Details of the preparation can be found elsewhere (Ringach et al. 1997a).

An increase in the firing rate of a neuron, as measured extracellularly, will be termed an "enhancement" of the neuron's response relative to some baseline. Similarly, a decrease in the firing rate of a neuron will be termed a "suppression" of the neuron's response. Enhancement and suppression are also often called "net excitation" and "net inhibition." We do this to avoid confusion with the words inhibition and excitation, which indicate, respectively, changes in the opening times of the inhibitory and excitatory ionic channels of a neuron. It is not possible to determine the underlying inhibitory and excitatory components of a cell uniquely from its extracellular firing rate. For example, response enhancement could be due to increased excitation, decreased inhibition, or a combination of both.

We measured the dynamics of tuning in the orientation and spatial frequency plane using a newly developed variant of the reverse correlation technique (Mazer et al. 2000; Ringach et al. 1997a,b). The stimulus is a sequence of luminance-modulated gratings with randomized orientations, spatial frequencies, and spatial phases presented rapidly one after the other at an effective rate of $50 \mathrm{~Hz}$ (Fig. 1A). The refresh rate of the monitor was $100 \mathrm{~Hz}$, but each image in the sequence was presented twice. The linear size of the stimulus was 1.5 to 3 times that of the classical receptive field, defined by the peak (or saturation point) of an area summation curve obtained with a grafting drifting at the optimal spatiotemporal parameters. The contrast of the stimulus was $99 \%$. The total experimental time was $15 \mathrm{~min}$.
The neuron's response consists of the arrival times of action potentials elicited during the period of visual stimulation. Given a fixed time lag, $\tau$, we calculate the probability that a grating of a particular spatial frequency, $\omega$, and orientation, $\theta$, preceded an impulse response by $\tau \mathrm{ms}, \operatorname{Pr}\{\omega, \theta ; \tau\}$. Spatial phase information is averaged in this computation. The result of the analysis is a family of two-dimensional probability distributions, one for each value of $\tau$. We investigate the dynamics of tuning by studying the evolution of the probability distribution with time. For small or large values of $\tau$ (usually for $\tau<$ $30 \mathrm{~ms}$ or $\tau>150 \mathrm{~ms}$ ), the stimulus does not influence the neuron's response and $\operatorname{Pr}\{\omega, \theta ; \tau\}$ approximates a uniform (flat) distribution. At intermediate values of $\tau$, the preference of a cell for a particular set of stimulus parameters is revealed as a peak in the probability distribution. Similarly, suppression of the cell's response for some stimuli produces valleys in the probability distribution. It should be noted that this "reverse correlation" method is formally equivalent to calculating the probability that a grating with a particular spatial frequency and orientation evokes a spike $\tau \mathrm{ms}$ after its presentation, or a "forward correlation" analysis.

Each image in the stimulus set was a Hartley basis function (Ringach et al. 1997b) of size $M \times M$ pixels, $H_{k_{x} k_{y}}(l, m)=$ $\operatorname{cas}\left\{\frac{2 \pi\left(k_{x} l+k_{y} m\right)}{M}\right\}$ for all $0 \leq 1, m \leq M-1$. Here, cas $\alpha \equiv \sin \alpha$ $+\cos \alpha$, and $k_{\mathrm{x}}$ and $k_{\mathrm{y}}$ represent the spatial frequency of the grating in units of cycles per stimulus side. The stimulus space consisted of all the basis functions $\left\{ \pm H_{k_{\mathrm{x}}, k_{\mathrm{y}}}\right\}$ such that $\left|k_{\mathrm{x}}\right| \leq k_{\max }$ and $\left|k_{\mathrm{y}}\right| \leq k_{\max }$. The maximal spatial frequency tested along the $x$ or $y$ axes is given by $\omega_{\max }=k_{\max } / L$, where $L$ is the size of one side of the stimulus patch in degrees of visual angle.

In defining our stimulus space, $k_{\max }$ was chosen to ensure that stimuli along the "boundary" of the space (given by all gratings in the set $\left.B=\left\{H_{k_{\mathrm{x}}, \pm \omega_{\max }} \vee H_{ \pm \omega_{\max }, k_{\mathrm{y}}}\right\}\right)$ had no effect on the neuron's response. The choice of $k_{\max }$ was based on the spatial frequency tuning for the cell to drifting sinusoidal gratings at the optimal

A

Stimulus

$\bullet$
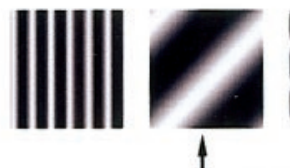

ENIIIVI/

Response

$\tau$

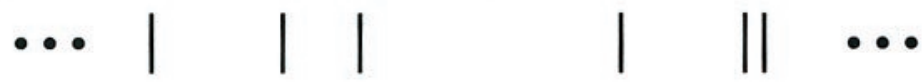

FIG. 1. A: segment of stimulus sequence and the response of the neuron. Each image in the stimulus sequence is a sinusoidal grating at different orientations, spatial frequencies and spatial phases. $B$ : plot of $R(\theta, \omega)$ in the Fourier plane. Areas in red represent response enhancement, areas in blue represent response suppression, and neutral stimuli are shown in green. The baseline is calculated as the probability of stimuli at the boundary of the stimulus space. These are the stimuli located between the 2 concentric outlined squares in the figure and correspond to the gratings having maximal spatial frequency at each orientation. 
spatiotemporal frequency and size. From the definition, it is clear that four spatial phases, $90^{\circ}$ apart, are present for each combination of $(\omega, \theta)$. We denote all gratings in the stimulus space by $S\left(k_{\max }\right)$. Each frame in the stimulus is drawn at random from $S\left(k_{\max }\right)$ with a uniform distribution.

To visualize the data, we compare the probability attained by each grating in the stimulus space to a baseline. A natural choice for the baseline is the probability of stimuli that are known to have no effect on the neuron's activity, such as sinusoidal gratings with arbitrary orientations but spatial frequencies so high that the cell cannot resolve them. We define the baseline $B(\tau)$ as the median probability of a set of such gratings. In our case, we selected stimuli located at the boundary of our stimulus space that correspond to the gratings with the highest spatial frequency tested at each orientation as defined by the set $B$ in the preceding text. The median was chosen to minimize the effects of a few responses reaching the boundary of the space, such as in Fig. $2 C$.

The empirical probability distributions were smoothed at each time using a $3 \times 3$ Gaussian kernel with $\sigma=1$ cycle/stimulus side and then transformed by calculating

$$
R(\omega, \theta ; \tau) \equiv \log _{10}\left\{\frac{\operatorname{Pr}\{\omega, \theta ; \tau\}}{B(\tau)}\right\}
$$

By definition, stimuli whose probability is the same as that of the baseline are mapped to $R(\omega, \theta ; \tau)=0$. Similarly, stimuli that enhance the response of the cell are mapped to values $R(\omega, \theta ; \tau)>0$, while stimuli that suppress the response of the cell are mapped to values $R(\omega, \theta ; \tau)<0$. There is an optimal time lag, $\tau_{\text {peak }}$, at which $R(\omega, \theta$; $\tau)$ reaches a maximum absolute value. The analysis in this study is based solely on $R(\omega, \theta) \equiv R\left(\omega, \theta ; \tau_{\text {peak }}\right)$. A description of the temporal dynamics of response enhancement and suppression will be the subject of a separate report.

$R(\omega, \theta)$ can also be interpreted as the log probability of finding a spike in a small time window $\tau_{\text {peak }}$ ms after a grating with parameters $(\omega, \theta)$. Using logarithms means that we consider enhancement and suppression equal in magnitude if the geometric mean of their firing probabilities equals the baseline probability. It can be shown that, under the assumption of Poisson firing, this definition implies that enhancement and suppression have equal magnitudes if and only if they can be discriminated equally well from the baseline rate of the cell (i.e., they have the same $d^{\prime}$, see APPENDIX).

To plot the results, we image $R(\omega, \theta)$ using a pseudo-color map (Fig. $1 B$ ). Areas of enhancement are represented by red hues, areas of suppression are represented by blue hues, and green areas represent neutral stimuli. The data are plotted in the Fourier plane (cf. DeValois

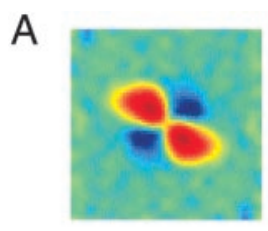

C

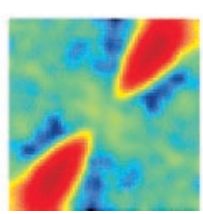

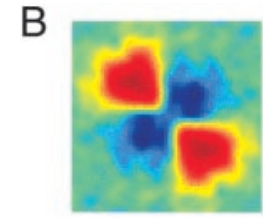

D

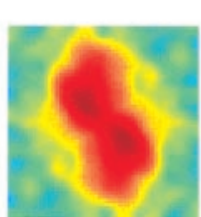

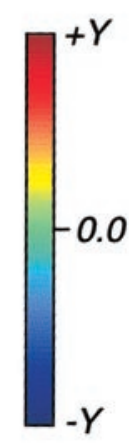

FIG. 2. Response enhancement and suppression in the Fourier plane. Each plot illustrates an example of $R(\theta, \omega)$. A-C: both enhancement by optimal stimuli and suppression by nonoptimal stimuli are evident in the majority of V1 cells. $D$ : some fraction of cells, like the one shown here, showed no evident suppression. The maximal spatial frequency along the $x$ axis and the amplitude of the scale, $Y$, for each case is as follows: 9.1 cycles/deg, $Y=1.35(A), 10.1$ cycles/deg, $Y=0.89$ $(B), 5.0$ cycles/deg, $Y=1.64(C)$, and 7.8 cycles/deg, $Y=1.2(D)$. et al. 1982; Jones et al. 1987). The origin is at the center of the graph. The distance from the origin to any point in the plane represents the spatial frequency of the grating at that location, and the angle with respect to the positive $x$ axis represents its orientation; the $x$ axis represents the spatial frequency of the grating in the horizontal meridian, $\omega_{x}=\omega \cos \theta$, and the $y$ axis represents the spatial frequency along the vertical meridian, $\omega_{y}=\omega \sin \theta$. All the relevant information is contained in the first and fourth quadrants: the data in the second and third quadrants are obtained by symmetry. We plot all four quadrants for visualization purposes only. Enhancement in the response of a cell is defined as significant if

$$
\max _{\omega, \theta, \tau} R(\omega, \theta ; \tau) \geq 4 \sqrt{\operatorname{var}(R(\omega, \theta ; 0))}
$$

That is, there is a peak in the response at least four times the SD of the noise. The same criterion was applied to determine the presence of a significant suppression after the appropriate sign change. Cells were subdivided into two sets. Cells that had both significant response enhancement and suppression are members of $S$. Cells with only significant enhancement are members of $\bar{S}$. Two cells had only a significant suppressive component and they were discarded.

\section{Computer simulations}

We simulated the reverse correlation experiment on three models of simple cortical neurons. First, a model cell whose membrane voltage depended linearly on the luminance contrast of the stimulus followed by half-wave rectification due to spike generation (Fig. 6C). The linear receptive field was modeled as a spatiotemporal separable function, $h(x, y, t)=A(x, y) B(t)$. The space kernel, $A(x, y)$, was a Gabor function: $A(x, y)=\exp \left(-x^{2} / 2 \sigma_{x}^{2}-y^{2} / 2 \sigma_{y}^{2}\right) \cos (2 \pi \omega x+\phi)$, and the temporal profile was a Hanning window, $B(n \Delta t)=1 / 2-\cos$ $[2 \pi n /(N+1)] / 2$, where $\Delta t=10 \mathrm{~ms}$ is the duration of one time slice and $n=1, \ldots, 8(n=8)$. Spatial kernels were sampled on a $64 \times 64$ grid representing $1 \times 1 \mathrm{deg}$ of visual angle. Two different types of spatial profiles were simulated: a filter that had broad tuning in orientation and spatial frequency and was even symmetric in space (Fig. 6A) and an odd-symmetric filter that was well tuned in orientation and spatial frequency (Fig. 6B). The parameters for the first filter were $\sigma_{x}=0.15 \mathrm{deg}, \sigma_{y}=0.15 \mathrm{deg}, \omega=1.6 \mathrm{cycles} / \mathrm{deg}$, and $\phi=0$ $\mathrm{deg}$, and for the second filter they were $\sigma_{x}=0.11 \mathrm{deg}, \sigma_{y}=0.25 \mathrm{deg}$, $\omega=5.3$ cycles $/ \mathrm{deg}$, and $\phi=90 \mathrm{deg}$. The static nonlinearity was a half-wave rectifier, $\Phi(x)=x$ if $x \geq 0$, and zero otherwise. This type of linear-nonlinear model is commonly used to model simple cells under constant levels of contrast gain control (Movshon et al. 1978).

We also explored two additional models that incorporate suppression. In the first of these models, a divisive gain control signal (Carandini et al. 1997), not tuned for orientation, and predominantly low-pass in spatial frequency was added to the linear-nonlinear model (Fig. 6D). The tuning of this feedback signal in the Fourier domain was equal to $F(\omega)=K \exp \left[-\left(\omega-\omega_{0}\right)^{2} / 2 \sigma_{\omega}^{2}\right]$, where $\omega_{0}=2$ cycles/deg and $\sigma_{\omega}=1.4$ cycles $/$ deg. Note that $F(\omega)$ is independent of the orientation, and therefore the feedback signal is untuned for orientation. In this model, the output of the linear receptive field is divided by a factor (the gain) that is proportional to the energy (variance) of the stimulus in a Fourier space weighted by $F(\omega)$. This value can be estimated by pooling the responses of cortical cells with different tuning selectivities. A purely feed-forward circuit can estimate the signal energy as well (Fig. 7). The temporal weights of the gain signal were identical to the temporal kernel of the linear filter. The second variant of a suppressive model used subtractive inhibition (Fig. $6 E$ ). Here, the output from a linear filter representing the excitatory input to the neuron is shifted by a DC hyperpolarization caused by a nonlinear suppressive signal. The magnitude of this hyperpolarization was proportional to the energy of the stimulus in the Fourier domain weighted by $F(\omega)$, which can also be estimated via pooling of cortical responses. This model may also be implemented in a feed- 
forward fashion (Fig. $6 F$ ). In both suppressive models, the tuning of the inhibitory signal in the Fourier plane overlapped partially with the tuning of the excitatory input but was broader in orientation and mostly low-pass in spatial frequency.

\section{R E S U L T S}

\section{Measurement of enhancement and suppression}

Response enhancement and suppression in different locations of the Fourier plane are evident in the majority of V1 neurons (Fig. 2). A typical example of $R(\omega, \theta)$ is shown in Fig. $2 A$. The magnitude of peak enhancement equals $R_{\max }=$ +1.29 . Thus at the peak time lag, the optimal stimulus is $\approx 20$ times more likely to precede a spike than baseline stimuli (see Eq. 1). Suppression has a similar magnitude of $R_{\min }=-1.35$. Nonoptimal stimuli are $\approx 22$ times less likely to precede a spike than baseline stimuli. A similar response profile is illustrated in Fig. 2B. Suppression may also peak at orientations flanking the optimal for the cell (Fig. 2C). Maximal response suppression occurs for stimuli about $30 \mathrm{deg}$ away from the optimal. This sort of flanking suppression in the orientation domain is consistent with our previous results on the dynamics of orientation tuning (Ringach et al. 1997a). Because a baseline was not available in previous studies, we could not establish with certainty that the valleys were the product of suppression. The present method overcomes this limitation and shows that suppression is indeed present in the responses of many cells.

There are some neurons that do not show response suppression (Fig. 2D). These cells tend to be broadly tuned for orientation and low-pass in spatial frequency. Such cells make up a significant proportion of our population $(31 / 75,41.3 \%)$, but the majority of cells had both response enhancement and suppression in their responses $(42 / 75,56 \%)$. Two cells had pure suppressive responses $(2 / 75,2.7 \%)$.

Our population consisted of 51 simple cells and 24 complex cells, defined as in Skottun et al. (1991). At present we do not observe any obvious differences between simple and complex cells, but a larger sample is required for a careful comparison of simple/complex properties.

To evaluate the relative importance of suppression in the development of tuning selectivity, we first compare the absolute magnitude of response enhancement and suppression in the subpopulation of neurons having both components (Fig. $3 A$ ). On average, peak suppression tends to be slightly smaller than enhancement. In a significant fraction of cells, however, enhancement and suppression have similar magnitudes. For such neurons, not responding to the "wrong" stimulus appears to be as important as responding to the "right" one.

Further insight into the role of suppression can be gained by looking at its location in the Fourier plane relative to the preferred stimulus for the cell (Fig. 3B). Here, data are normalized so that the peak response enhancement for each cell is always located at zero orientation and a spatial frequency of one [that is, at $\left.\left(\omega_{\mathrm{x}}, \omega_{\mathrm{y}}\right)=(1,0)\right]$. The distance from the origin represents the ratio between the spatial frequency for peak suppression and enhancement. The $x$ axis represents the horizontal component of the spatial frequency and the $y$ axis the vertical component. Points inside the circular sector of radius one have peak suppression at spatial frequencies lower than that of enhancement. The angle between each point and the positive $x$ axis represents the relative difference in orientation
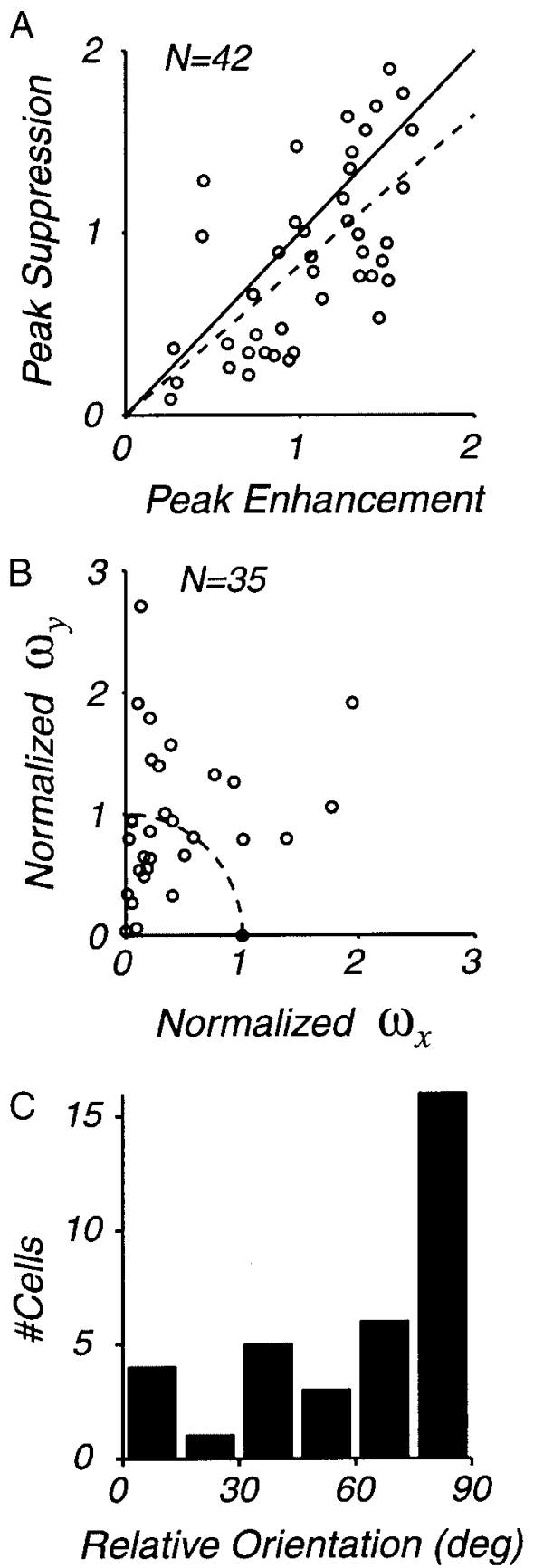

FIG. 3. A: scatter-plot of the magnitude of peak enhancement vs. magnitude of peak suppression. $B$ : location of suppression relative to enhancement. The data were normalized so that the location of peak enhancement occurs at $(x, y)=(1,0)$. The distance from the origin represents the ratio between the optimal spatial frequency for suppression and inhibition. The angle with respect to the positive $x$ axis indicates the location of suppression relative to enhancement. Cells with preferences for very low spatial frequencies are excluded from this analysis as the estimation of angles between enhancement and suppression is unreliable in these cases. $C$ : histogram of the relative angle between peak suppression relative to peak enhancement. There is a tendency for suppression to occur at an angle orthogonal to that of enhancement.

between the location of peak enhancement and suppression in the Fourier plane. Peak suppression tends to occur at orientations near the orthogonal to that of peak enhancement and for similar spatial frequencies. However, cases where suppression peaks at oblique angles are also observed (Fig. $3 C$ ). In about 
$80 \%$ of the cases, the spatial frequency of suppression is within an octave of the optimum for response enhancement.

\section{Suppression and orientation selectivity}

The comparable magnitudes of peak enhancement and suppression and the particular location of suppression in the Fourier plane suggest that suppression of neural responses to nonoptimal stimuli may play an important role in the development of neural selectivity. Therefore we looked for a correlation between the presence or absence of suppression in the responses of neurons and their degree of selectivity. If the hypothesis is correct, one would expect cells that are suppressed by nonoptimal stimuli to have higher selectivity than neurons with purely excitatory responses. Indeed, we find that selectivity for stimulus orientation is higher in neurons whose responses exhibit suppression for nonoptimal stimuli than for cells that do not. To obtain this result, we first divide our population into two groups: cells that have a significant suppressive component in their response, $S(n=42)$ and those that do not, $\bar{S}$ ( $n=31$; see METHODS). Then we ask if orientation selectivity is higher for cells in $S$ compared with those in $\bar{S}$. To cross-validate results, we calculated the orientation selectivity of neurons based on an analysis of the dynamics data as well as the response of the cell to sinusoidal gratings drifting at a constant speed. The first measure is derived from the dynamics measurements by calculating the angular projection of the response inside a disk in Fourier space:

$$
R(\theta)=\int_{\omega \leq \omega_{\max }} R^{+}(\omega, \theta) \mathrm{d} \omega
$$

Here, $R^{+}(\omega, \theta)$ represents the positive part of $R(\theta, \omega)$; the negative part is clipped to zero. Averaging over spatial frequencies increases the signal to noise of our measurements and is reasonable given that most of the responses are very well approximated by separable functions in spatial frequency and orientation. The circular variance of the projection gives a measure of orientation tuning selectivity and is defined as (Mardia 1972)

$$
V=1-\frac{\left|\int R(\theta) e^{\mathrm{i} 2 \theta} \mathrm{d} \theta\right|}{\int R(\theta) \mathrm{d} \theta}
$$

This measure is bounded between zero and one. Cells that are highly selective for stimulus orientations have circular variance values close to zero. Cells that have broad selectivity are mapped to values close to one.

The distribution of the circular variance of $R(\theta)$, for both cell groups, is shown in Fig. $4 A$. Cells that have a suppressive component in their dynamics are more selective (have lower circular variance) than those that do not $\left(P<1.5 \times 10^{-8}\right.$, Wilcoxon rank sum test with continuity correction). By definition, the way we calculated circular variance as a measure of orientation selectivity only reflects the tuning of response enhancement [due to the positive clipping of $R(\theta, \omega)]$. Thus there is no reason why, in principle, selectivity and suppression should be correlated with one another, but we find that they are.

Also we evaluated the orientation selectivity of a neuron
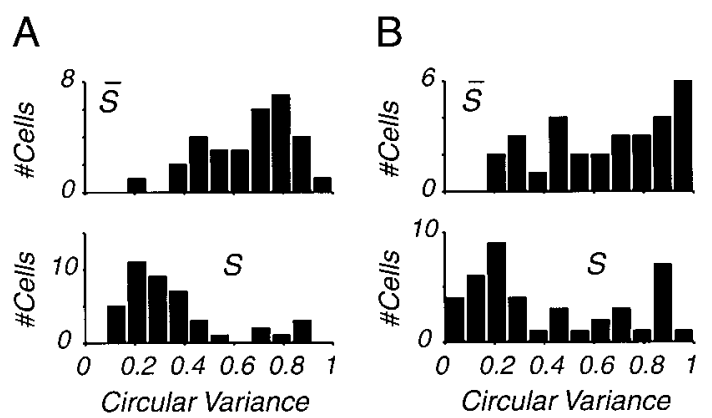

FIG. 4. Suppression for nonoptimal stimuli correlates with orientation selectivity in V1 neurons. A: histogram of circular variance calculated from dynamics data in cells with $(S)$ and without $(\bar{S})$ suppressive components. $B$ : distribution of circular variance calculated based on the steady-state orientation tuning curves.

based on its steady-state tuning curve. In this experiment, we measure the steady-state orientation tuning curve of a cell using drifting gratings at its optimal spatiotemporal frequency and define $R(\theta)$ as the mean firing rate at each drift direction. The circular variance of $R(\theta)$ is calculated from the steady-state tuning curve using the same formula as above. Histograms of the circular variance of $R(\theta)$, for both cell groups, are illustrated in Fig. 4B. Steady-state selectivity is also significantly higher for cells showing suppression for nonoptimal stimuli than those showing pure excitatory responses $\left(P<7 \times 10^{-4}\right.$, Wilcoxon rank sum test with continuity correction).

\section{Suppression and spatial frequency selectivity}

Spatial frequency tuning is also influenced by suppression. The main effect of suppression is in shaping the low-frequency limb of the tuning curve. Cells with pure excitatory components tend to have low-pass tuning curves, while cells that show suppression tend to have band-pass tuning curves. To obtain this result, we first use the dynamic measurements to calculate the average response at each spatial frequency averaged across all orientations,

$$
R(\omega)=\frac{1}{2 \pi \omega} \int_{\theta} R^{+}(\omega, \theta) \mathrm{d} \theta
$$

The ratio between the response at the lowest spatial frequency tested $\left(\omega_{0}\right)$ and the response at the peak spatial frequency $\left(\omega_{\text {peak }}\right), \alpha \equiv R\left(\omega_{0}\right) / R\left(\omega_{\text {peak }}\right)$, provides an index that is close to zero for tuning curves that are band-pass shaped and close to one for tuning curves that are low-pass shaped. We call $\alpha$ a low-pass index. Histograms of $\alpha$, for both cell groups, are illustrated in Fig. 5A. Cells that have a suppressive component in their dynamics have lower low-pass indices (tuning curves that are more band-pass shaped) than those that exhibit pure excitatory components in their responses $\left(P<2 \times 10^{-6}\right.$, Wilcoxon rank sum test with continuity correction).

A similar result is obtained if we analyze the steady-state spatial frequency tuning curves. Here, $R(\theta)$ represents the mean firing rate at each spatial frequency. Histograms of $\alpha$, for both cell groups, are illustrated in Fig. $5 B$. Cells showing suppression for nonoptimal stimuli have lower low-pass indices (i.e., the tuning curves are more band-pass) than those showing pure excitatory responses $\left(P<7 \times 10^{-3}\right.$, Wilcoxon rank sum test with continuity correction). 
A
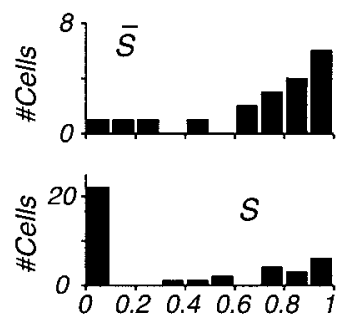

Low-pass index
B
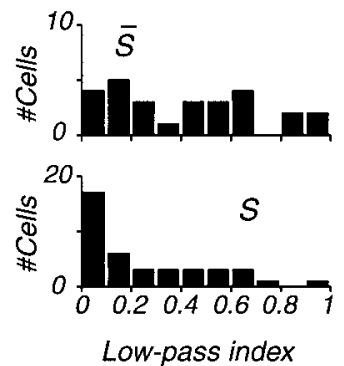

FIG. 5. Suppression for nonoptimal stimuli correlates with the shape of spatial frequency tuning curves in V1 neurons. A: histogram of low-pass indices calculated from dynamics data in cells with $(S)$ and without $(\bar{S})$ suppressive components. $B$ : distribution of low-pass indices calculated based on the steady-state spatial frequency tuning curves.

\section{Comparison with models}

We compared the experimental results with the prediction of three mathematical models. First, we simulated the response of a quasi-linear feedforward model to our stimulus (Fig. 6C) (Movshon et al. 1978). The model consists of a linear spatiotemporal filter followed by half-wave rectification. The input is the luminance contrast spatiotemporal pattern generated by the stimulus and the output is the rate of firing of the cell. In the APPENDIX, we formally show that if the static nonlinearity is convex (as in the case of a half-rectifier or half-squaring) the resulting kernels will always be positive (independently of the form of the feedforward filter). This means that such a model cannot generate suppression as defined by our analysis. The simulations confirm this theoretical result.

We offer two examples of feed-forward receptive fields. In both cases, the linear receptive field was modeled as a separable filter in space and time. In the first example, the space kernel was an even-symmetric Gabor function broadly tuned in orientation and low-pass in spatial frequency, denoted by $h_{1}$ (Fig. 6A). In the second case (Fig. 6B), the filter was an odd-symmetric Gabor function well tuned in both orientation and spatial frequency, denoted by $h_{2}$. The reverse correlation kernels obtained for the different models are shown as panels in Fig. 6, C-E. The left panel corresponds to the results of $h_{1}$ and the right panels correspond to $h_{2}$.

As expected, the results of the simulation for the linearnonlinear model reveal responses with no suppressive component (Fig. 6C). In this sort of feedforward model, tuning selectivity can be set to high or low by an appropriate choice of the linear kernel. The kernel obtained using $h_{1}$, shown in the left panel of Fig. $6 C$, has broad tuning (circular variance of 0.8 and low-pass index of 0.8). In contrast, the kernel generated by $h_{2}$ has sharp selectivity in the Fourier plane (circular variance of 0.25 and low-pass index of 0.1). However, because the linear-nonlinear model never generates suppression, it cannot explain the correlation between suppression and tuning in our data. The linear-nonlinear model could account for neurons like the one whose results are shown in Fig. $2 D$. Such weakly tuned neurons comprise a significant fraction of the data set.

A nonlinear suppressive signal is therefore required to explain the results in the sharply tuned neurons. We then investigated if the addition of suppression to the basic linear-nonlinear model could sharpen tuning selectivity. We considered two different suppression mechanisms: divisive and subtrac-

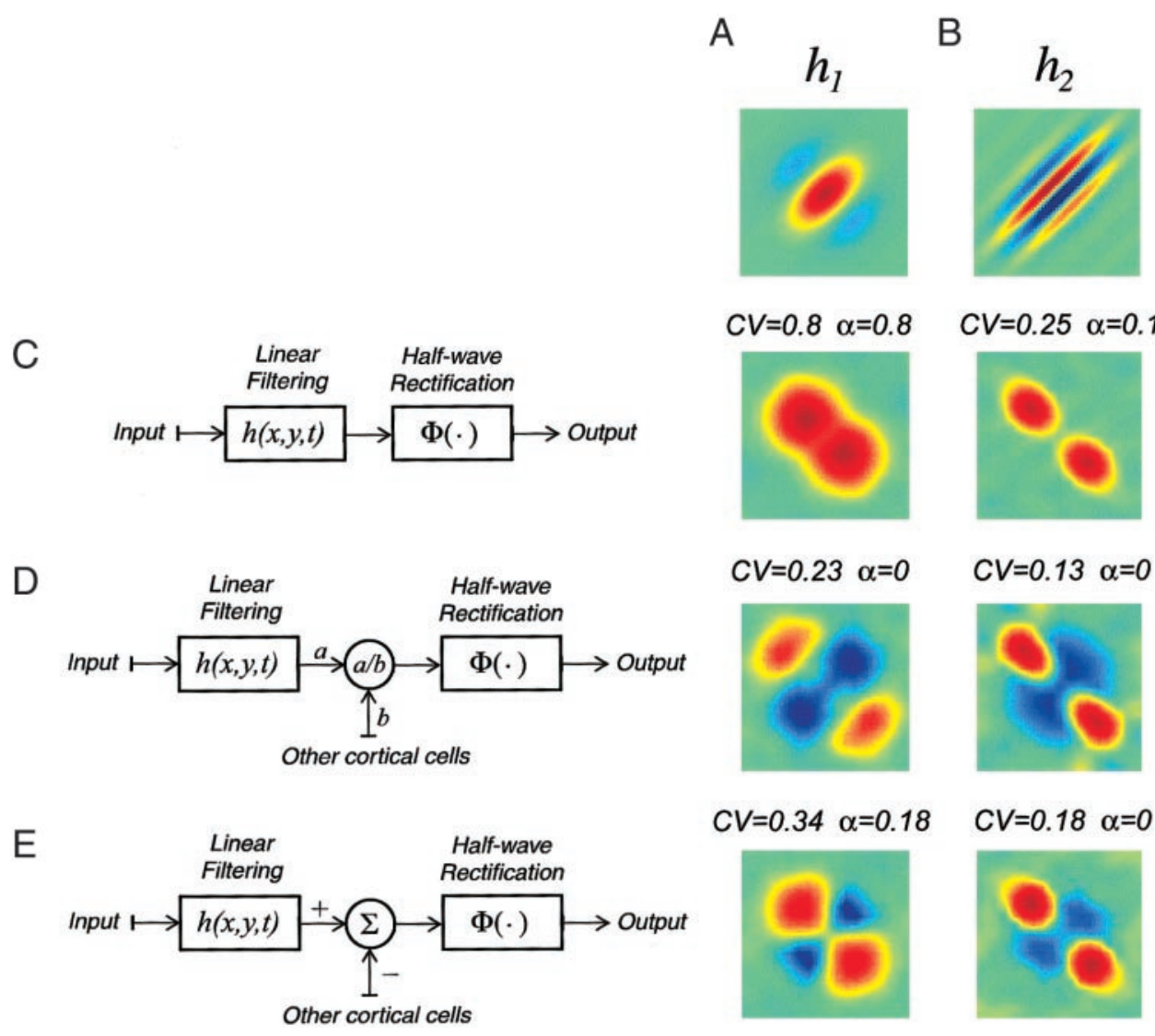

FIG. 6. Computer simulation. $A$ and $B$ : the two spatial kernels used in the simulations, $h_{1}$ and $h_{2}$. $C$ : a linear-nonlinear cascade model of simple cell function. The two kernels depict the result of simulating the reverse correlation mapping experiment on this model for both $h_{1}$ (left) and $h_{2}(r i g h t)$. D: a divisive inhibition model and the resulting kernels from the simulation. $E$ : a subtractive inhibition model and the resulting kernels from the simulation. The circular variance and low-pass index for each kernel are written on top of each panel. 
tive. In the models we studied, suppression is assumed to originate as a feedback signal in the cortex by pooling a number of cortical cells, but as we discuss in the following text, equivalent feed-forward implementations can be postulated as well. Thus while our data show that suppression might be important in the generation of tuning they do not speak as to the structure of the circuit underlying its generation.

In the divisive suppression scheme, the output of the linear filter is divided by a gain signal obtained by pooling the activities of a large number of cortical cells with different tuning selectivities (e.g., Carandini et al. 1997). The resulting kernel in the left panel of Fig. $6 D$ resembles some of the empirical kernels (Fig. 2, $A$ and $B$ ) and shows that divisive inhibition can be used to enhance selectivity. For $h_{1}$, divisive inhibition generates a kernel with $\mathrm{CV}=0.23$ and $\alpha=0$, compared with the linear-nonlinear model, which has $\mathrm{CV}=$ 0.8 and $\alpha=0.8$ ). A similar outcome is obtained if we use subtractive inhibition (Fig. 6E). Here suppression acts by shifting the feedforward contribution away from threshold. These simulations demonstrate that both subtractive or divisive feedback can transform a broadly tuned excitatory component (Fig. $6 C$, left) into a response with higher selectivity in orientation and spatial frequency (Fig. 6, D and E, left). Adding suppression to a well-tuned filter generates similar kernels but the sharpening effect is much reduced (Fig. 6, $C-E$, right). These findings, together with the fact that we rarely observe sharply tuned cells without suppression (as in the right panel in Fig. $6 C$ ), are consistent with the view that feedforward mechanisms may provide broad stimulus selectivity to the cortex, but the way the cortex seems to achieve high selectivity is by the use of intracortical inhibition.

Based solely on the qualitative shapes of the simulated kernels, it appears that our data cannot distinguish between divisive and subtractive inhibition. We are now investigating if a quantitative comparison of the resulting kernels may provide a way to differentiate between the two. Recent intracellular experiments in cat visual cortex, however, suggest that shunting (divisive) inhibition plays an important role in suppressing nonoptimal responses (Monier et al. 2000; but see Anderson et al. 2000; Ferster et al. 1996).

\section{I S C U S S I O N}

We used a reverse correlation technique to map response enhancement and suppression in V1 neurons. The method can be considered a refinement of the classical "conditioning stimulus" technique, where the response to a test stimulus is measured on top of some elevated firing rate set by a conditioning (or base) stimulus (e.g., among others, Blakemore and Tobin 1972; Bonds 1989; Nelson and Frost 1978). This elevated rate facilitates the detection of suppression. In the reverse correlation method, the elevation in firing rate is caused by the rapid stimulus sequence itself. The elements in the sequence are themselves the test stimuli, which appear multiple times embedded in different contexts. The analysis averages the effect of a test stimulus across the different contexts. A significant advantage of the reverse correlation technique is that the cortex does not adapt to the "base" stimulus (as is the case in the conditioning paradigm). This is a potential problem with the classical conditioning stimulus technique because it is known that adaptation can induce changes in tuning (Dragoi et al.
2001). An additional refinement we have made in the method over our previous work (Ringach et al. 1997b) is to define a baseline response explicitly. Suppression can now be measured directly instead of being inferred from the "Mexican-hat" shape of the tuning functions. Furthermore, global (uniform) suppression, which might have gone undetected by the previous method, can now be measured as well. Finally, by measuring response on the whole Fourier domain with reverse correlation, we have obtained a clear picture of the relationship between enhancement and suppression in V1.

Previous work showed the presence of response suppression in the orientation or spatial frequency domains but did not systematically study the relationship between suppression and selectivity or make comparisons to model outputs (Bauman and Bonds 1991; Blakemore and Tobin 1972; Bonds 1989; Burr et al. 1981; De Valois and Tootell 1983; De Valois et al. 1982; Morrone et al. 1982; Nelson and Frost 1978; Nelson 1991; Ringach et al. 1997a; Sclar and Freeman 1982). By using a new technique that allows us to map simultaneously the precise location of response enhancement and suppression in the Fourier plane, we found that suppression and tuning selectivity are correlated in V1 neurons. Cells that are suppressed by nonoptimal stimuli are more selective, both in the orientation and spatial frequency domains. In the spatial frequency domain, however, suppression acts mainly on the lower-frequency-limb of the tuning curve, enhancing the neurons' bandpass characteristics. This was established without the need for pharmacological manipulations as done in some previous studies. Instead we took advantage of the natural variability of tuning selectivity in the cortex to study the correlation between inhibition and tuning selectivity in the normal V1 population.

The modeling shows that a broadly tuned inhibition, which overlaps significantly with the response of the feedforward signal in the Fourier domain, is sufficient to generate results that are qualitatively similar with most of the experimental data (a tuned inhibitory signal is required to replicate the flank suppression in Fig. 2C). Broadly tuned suppression was used in the simulations to be consistent with experimental results in cat area 17 (DeAngelis et al. 1992), which show a suppressive component that is broadly tuned for orientation and spatial frequency. Both subtractive and divisive inhibition generated results that are in qualitative agreement with the measured kernels. The divisive model has been proposed before to account for contrast gain control in the cortex (Albrecht and Geisler 1991; Carandini et al. 1997; Heeger 1992). The suppression observed in the kernels could be related to a contrast gain control signal acting on fast time scales. An assumption of these models is that response selectivity is invariant at different levels of contrast gain control. However, the observed correlation between selectivity and suppression, together with the modeling results and the findings of previous studies (McLaughlin et al. 2000), show that in addition to controlling contrast gain, divisive inhibition might sharpen response selectivity in the cortex.

A question that remains unanswered in our study is the exact structure of the cortical circuitry generating the inhibitory signals. The numerical simulations show that cortical feedback is one possibility. In principle, feedback suppression can be replaced with feed-forward suppression using a circuit as shown in Fig. 7A. Here, the nonlinear suppressive signal is generated by summing in a feed-forward fashion the output of 


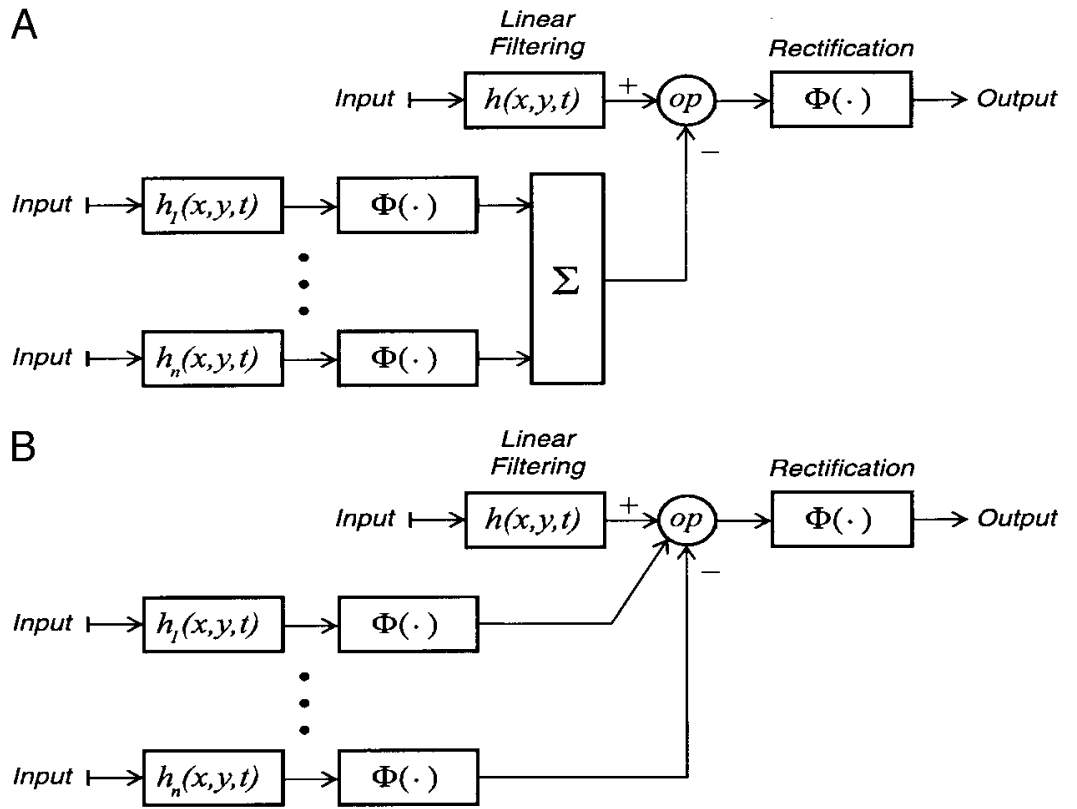

FIG. 7. Feed-forward implementations of the models in Fig. 6 ( $D$ and $E$ ). Op is an operator that can be either subtraction or division. $A$ : the output from several simple cells with different preferences in the Fourier domain are pooled to generate a complex-like cell that provides the required (nonlinear) suppressive signal. $B$ : the output from several (inhibitory) simple cells with different preferences in the Fourier domain synapse directly onto the target cell.

simple cells. This is similar to the hierarchical complex cell model of Hubel and Wiesel. The nonlinear signal is then used to inhibit, via division or subtraction, the target cell. An alternative is that several inhibitory simple-cells synapse directly on the target cell (Fig. 7B). These feed-forward inhibition models can be made to generate results that resemble those of the feedback models.

Because there is no evidence for direct thalamo-cortical inhibition, the feed-forward inhibition models postulate the existence of a cortical inhibitory interneuron for the main purpose of inverting the LGN input (Troyer et al. 1998). However, we feel the existence of such purely "feed-forward" circuitry through inhibitory interneurons is doubtful given our knowledge of the cortical anatomy (Dantzker and Callaway 2000; Lund 1987, 1988; Lund et al. 1995). It appears likely that inhibitory interneurons also receive a substantial cortical input that is dependent on visual stimulation and that the entire circuit is more appropriately described as a feedback system. Nevertheless the actual organization of the inhibitory circuitry remains a question for further research.

The data presented here significantly advance our knowledge of the relationship between suppression and tuning. Specifically, the correlations in our data are consistent with the idea that nonlinear suppression, whether generated by feedback or feed-forward mechanisms, plays a role in generating sharp stimulus selectivity in the Fourier plane. Models of cortical selectivity need to predict the observed correlation between response suppression and tuning selectivity in primary visual cortex. Simple models that generate high orientation selectivity through feed-forward (linear) excitatory mechanisms but no suppression, as shown by simulations (Fig. 6) and analytically, do not predict such a correlation and therefore are rejected by the data.

\section{A P P E N D I X}

\section{Calculation of $d^{\prime}$ for the Poisson case}

Given two Poisson distributions, with rates $\lambda$ and $\alpha \lambda$, we can derive a measure of $d^{\prime}$ analogous to the Gaussian case (Green and Swets
1966). The parameter $d^{\prime}$ gives a measure of how separated the two distributions are-the more separated the rates are the higher the $d^{\prime}$. The log-likelihood function for a Poisson distributed variable is easily calculated and equals $L(k)=-\lambda+k \ln \lambda-\ln k$ !. Thus the $\log$-likelihood ratio between two hypotheses with rates $\lambda$ and $\alpha \lambda$ can be written as, $l(k)=k \ln \alpha+C$, where $C$ is a constant that depends on $\lambda$ and $\alpha$. In analogy to the equal-variance Gaussian case, we can write $d^{\prime}=\ln \alpha$. The parameter $d^{\prime}$ is the $\log$ of the ratio between the means of the two distributions. Given a baseline rate $\lambda$ there are only two different rates that will yield the same (absolute) $d^{\prime}$ value: $\lambda e^{\mathrm{d}^{\prime}}$ and $\lambda e^{-\mathrm{d}^{\prime}}$. Clearly, the product of these rates equals $\lambda$, and the logarithm of the ratio between the rates and the baseline rate equals $\pm d^{\prime}$. Therefore the absolute magnitude of enhancement and suppression, as defined in this study, is effectively a measure of $d^{\prime}$ relative to the baseline rate.

\section{Nonnegativity of kernels in a linear-nonlinear system}

Consider the linear-nonlinear system depicted in Fig. $6 C$. We claim that if $\Phi(\cdot)$ is convex then the reverse correlation kernel is nonnegative, $R(\omega, \theta ; \tau) \geq 0$.

In other words, this model cannot generate suppression as defined in our analysis. This can be seen as follows. Let us denote by $y(t)$ the output of the linear filter at time $t$ and the instantaneous (Poisson) rate of firing by $r(t)=\Phi[y(t)]$. At any point in time, the probability of the neuron firing in an interval $\Delta t$ is given $r(t) \Delta t$. Thus the expected firing rate $\tau \mathrm{ms}$ after the presentation of a grating with a fixed spatial frequency $(\omega)$ and orientation $(\theta)$, but random spatial phase $(\phi)$ is given by

$$
E\{\Phi(y)\}=E\{\Phi(H(\omega, \theta ; \tau) \sin \phi+z)\}
$$

Here, $H(\omega, \theta ; \tau)$ is the amplitude spectrum of the linear filter evaluated at $t=\tau$, and $z$ is a random variable that represents the contribution of the filter to $y(t)$ from times other than $\tau$. If $\Phi(\cdot)$ is convex, we can use Jensen's inequality

$$
E\{\Phi(y)\} \geq \Phi(E\{y\})
$$

to obtain

$$
E\{\Phi(H(\omega, \theta ; \tau) \sin \phi+z)\} \geq \Phi(E\{H(\omega, \theta ; \tau) \sin \phi+z\})
$$

but 


$$
\begin{aligned}
\Phi(E\{H(\omega, \theta ; \tau) \sin \phi+z\}) & =\Phi(E\{H(\omega, \theta ; \tau) \sin \phi\}+E\{z\}) \\
& =\Phi(E\{H(\omega, \theta ; \tau) \sin \phi\}) \\
& =\Phi(0)
\end{aligned}
$$

Which yields

$$
E\{\Phi(H(\omega, \theta ; \tau) \sin \phi+z)\} \geq \Phi(0)
$$

However, $\Phi(0)$ is also the firing rate of a baseline stimulus, as the definition requires that for such stimuli $H(\omega, \theta ; \tau)=0$, which means that the stimuli cannot be resolved by the neuron. We conclude that

$$
R(\omega, \theta ; \tau) \equiv \log _{10}\left\{\frac{E\{\Phi(H(\omega, \theta ; \tau) \sin \phi+z)\}}{\Phi(0)}\right\} \geq \log _{10}\{1\} \geq 0
$$

This research was supported by National Eye Institute Grants EY-12816 (D. L. Ringach), EY-08300 (M. J. Hawken), and EY-01472 (R. M. Shapley) and by the Sloan Foundation for support of the New York University Theoretical Neuroscience Program.

\section{REFERENCES}

ALBRECHT DG AND GEISLER WS. Motion selectivity and the contrast-response function of simple cells in the visual cortex. Vis Neurosci 7: 531-546, 1991.

ALLISON JD AND Bonds AB. Inactivation of the infragranular striate cortex broadens orientation tuning of supragranular visual neurons in the cat. Exp Brain Res 101: 415-426, 1994.

Allison JD, Casagrande VA, AND Bonds AB. The influence of input from the lower cortical layers on the orientation tuning of upper layer V1 cells in a primate. Vis Neurosci 12: 309-320, 1995.

Allison JD, Kabara JF, Snider RK, Casagrande VA, and Bonds AB. $\mathrm{GABA}_{\mathrm{B}}$-receptor-mediated inhibition reduces the orientation selectivity of the sustained response of striate cortical neurons in cats. Vis Neurosci 13: 559-566, 1996.

ANDERSON JS, CARANDini M, AND FERSTER D. Orientation tuning of input conductance, excitation, and inhibition in cat primary visual cortex. $\mathrm{J} \mathrm{Neu}$ rophysiol 84: 909-926, 2000.

Azouz R, Gray C, NowaK L, AND MCCoRmick D. Physiological properties of inhibitory interneurons in cat striate cortex. Cereb Cortex 7: 534-545, 1997.

BAUMAN LA AND BondS AB. Inhibitory refinement of spatial frequency selectivity in single cells of the cat striate cortex. Vision Res 31: 933-944, 1991.

BEN-YISHAI R, BAR-OR RL, AND SOMPOLINSKY H. Theory of orientation tuning in visual cortex. Proc Natl Acad Sci USA 92: 3844-3848, 1995.

Benevento LA, CReutzFeldt OD, AND Kuhnt U. Significance of intracortical inhibition in the visual cortex. Nat New Biol 238: 124-126, 1972.

BLAKEMORE C AND TOBIN EA. Lateral inhibition between orientation detectors in the cat's visual cortex. Exp Brain Res 15: 439-440, 1972.

Bonds AB. Role of inhibition in the specification of orientation selectivity of cells in the cat striate cortex. Vis Neurosci 2: 41-55, 1989.

Burr D, Morrone C AND MAFFeI L. Intra-cortical inhibition prevents simple cells from responding to textured visual patterns. Exp Brain Res 43: 455458, 1981.

Carandini M and Ferster D. Membrane potential and firing rate in cat primary visual cortex. J Neurosci 20: 470-484, 2000.

CARANDINI M, HEEGER DJ, AND Movshon JA. Linearity and normalization in simple cells of the macaque primary visual cortex. J Neurosci 17: 86218644, 1997.

ChUng S AND Ferster D. Strength and orientation tuning of the thalamic input to simple cells revealed by electrically evoked cortical suppression. Neuron 20: 1177-1189, 1998.

Crook JM, Kisvarday ZF, AND Eysel UT. Evidence for a contribution of lateral inhibition to orientation tuning and direction selectivity in cat visual cortex: reversible inactivation of functionally characterized sites combined with neuroanatomical tracing techniques. Eur J Neurosci 10: 2056-2075, 1998.

DANTZKer JL AND CALlaway EM. Laminar sources of synaptic input to cortical inhibitory interneurons and pyramidal neurons. Nat Neurosci 3: 701-707, 2000.

De Angelis GC, Robson JG, Ozhawa I, And Freeman RD. Organization of suppression in receptive fields of neurons in cat visual cortex. $J$ Neurophysiol 68: 144-163, 1992.
DE VALOIS KK AND TOOTELL RB. Spatial-frequency-specific inhibition in cat striate cortex cells. J Physiol (Lond) 336: 359-376, 1983.

De Valois RL, Yund EW, and HePler N. The orientation and direction selectivity of cells in macaque visual cortex. Vision Res 22: 531-544, 1982.

DRAGoi V, Rivadulla C, AND SUR M. Foci of orientation plasticity in visual cortex. Nature 411: 80-86, 2001.

FERSTER D. Orientation selectivity of synaptic potentials in neurons of cat primary visual cortex. J Neurosci 6: 1284-1301, 1986.

Ferster D, Chung S, and Wheat H. Orientation selectivity of thalamic input to simple cells of cat visual cortex. Nature 380: 249-252, 1996.

FERSTER D AND Miller KD. Neural mechanisms of orientation selectivity in the visual cortex. Annu Rev Neurosci 23: 441-471, 2000.

Green DM and Swets JA. Signal Detection Theory and Psychophysics. Los Altos, CA: Peninsula, 1966.

Hata Y, Tsumoto T, Sato H, Hagihara K, and Tamura H. Inhibition contributes to orientation selectivity in visual cortex of cat. Nature 335: 815-817, 1988.

HEEGER DJ. Normalization of cell responses in cat striate cortex. Vis Neurosci 9: 181-197, 1992.

Hubel DH AND WIESEL TN. Receptive fields, binocular interaction and functional architecture in the cat's visual cortex. J Physiol (Lond) 160: 106-154, 1962.

Hubel DH AND WiESEL TN. Receptive fields and functional architecture of monkey striate cortex. J Physiol (Lond) 195: 215-243, 1968.

Jones JP, Stepnoski A, AND PALMER LA. The two-dimensional spectral structure of simple receptive fields in cat striate cortex. J Neurophysiol 58: 1212-1232, 1987.

LUND JS. Local circuit neurons of macaque monkey striate cortex. I. Neurons of laminae 4C and 5A. J Comp Neurol 257: 60-92, 1987.

LUND JS. Anatomical organization of macaque monkey striate visual cortex. Annu Rev Neurosci 11: 253-288, 1988.

Lund JS, Wu Q, Hadingham PT, and LevitT JB. Cells and circuits contributing to functional properties in area V1 of macaque monkey cerebral cortex: bases for neuroanatomically realistic models. J Anat 187: 563-581, 1995.

Mardia KV. Statistics of Directional Data. London: Academic, 1972.

Mazer JA, David SV, and Gallant JL. Spatiotemporal receptive field estimation during free viewing visual search in macaque striate and extrastriate cortex. Soc Neurosci Abstr 26: 141, 2000.

McLaughlin D, Shapley R, Shelley M, and Wielaard DJ. A neuronal network model of macaque primary visual cortex (V1): orientation selectivity and dynamics in the input layer $4 \mathrm{C} \alpha$. Proc Natl Acad Sci USA 97: 8087-8092, 2000.

Monier C, Chavane F, Baudot P, Borg-Graham L, and Fregnac Y. Direct evidence for a role of inhibition in orientation and direction selectivity in V1 neurons. Soc Neurosci Abstr 26: 139, 2000.

MorRone MC, BURR DC, AND MAFFEI L. Functional implications of crossorientation inhibition of cortical visual cells. I. Neurophysiological evidence. Proc R Soc Lond B Biol Sci 216: 335-354, 1982.

Movshon JA, ThOMPSON ID, AND TolhuRst DJ. Spatial summation in the receptive fields of simple cells in the cat's striate cortex. $J$ Physiol (Lond) 283: 53-77, 1978 .

Nelson JI AND Frost BJ. Orientation-selective inhibition from beyond the classic visual receptive field. Brain Res 139: 359-365, 1978.

NeLson SB. Temporal interactions in the cat visual system. I. Orientationselective suppression in the visual cortex. J Neurosci 11: 344-356, 1991.

Nelson S, Toth L, Sheth B, ANd Sur M. Orientation selectivity of cortical neurons during intracellular blockade of inhibition. Science 265: 774-777, 1994.

Nestares O AND HeEger DJ. Modeling the apparent frequency-specific suppression in simple cell responses. Vision Res 37: 1535-1543, 1997.

Pugh MC, Ringach DL, Shapley R, and Shelley MJ. Computational modeling of orientation tuning dynamics in monkey primary visual cortex. J Computat Neurosci 8: 143-159, 2000.

Ramoa AS, Shadlen M, Skottun BC, and Freeman RD. A comparison of inhibition in orientation and spatial frequency selectivity of cat visual cortex. Nature 321: 237-239, 1986.

RingaCh DL, HAWKEN MJ, AND ShaPLEY R. Dynamics of orientation tuning in macaque primary visual cortex. Nature 387: 281-284, 1997.

RingaCh DL, SAPIRO G, AND ShaPley R. A subspace reverse correlation method for the study of visual neurons. Vision Res 37: 2455-2464, 1997.

Sato H, Katsuyama N, Tamura H, Hata Y, and Tsumoto T. Mechanisms underlying orientation selectivity of neurons in the primary visual cortex of the macaque. J Physiol (Lond) 494: 757-771, 1996. 
Sillito AM, Kemp JA, Milson JA, AND Berardi N. A re-evaluation of the mechanisms underlying simple cell orientation selectivity. Brain Res 194: 517-520, 1980.

Skottun BC, De Valois RL, Grosof DH, Movshon JA, Albrecht DG, AND Bonds AB. Classifying simple and complex cells on the basis of response modulation. Vision Res 31: 1079-1086, 1991.

SOMERS DC, NELSON SB, AND SUR M. An emergent model of orientation selectivity in cat visual cortical simple cells. J Neurosci 15: 5448-5465, 1995 .
SOMPOLINSKY H AND SHAPLeY R. New perspectives on the mechanisms for orientation selectivity. Curr Opin Neurobiol 7: 514-522, 1997.

Troyer WT, Krukowski AE, Priebe NJ, AND Miller KD. Contrast-invariant orientation tuning in cat visual cortex: thalamocortical input tuning and correlation-based intracortical connectivity. J Neurosci 18: 5908-5927, 1998.

Volgushev M, Pei X, Vidyasagar TR, AND CReutzFeldt OD. Excitation and inhibition in orientation selectivity of cat visual cortex neurons revealed by whole-cell recordings in vivo. Vis Neurosci 10: 1151-1155, 1993. 